\title{
DXA Body Composition is Weakly Related to Blood Lipids, Blood Pressure, and Glucose in Firefighters
}

\author{
KALEN A. JOHNSON, ALLISON L. DONNELL, DANIEL K. MOHNKE, STEVEN E. \\ MARTIN, JOHN S. GREEN FACSM, STEPHEN CROUSE FACSM
}

Applied Exercise Science Laboratory; Department of Health and Kinesiology; Texas A\&M University; College Station, TX

Category: Masters

Advisor/Mentor: Crouse, Stephen (s-crouse@tamu.edu)

\section{ABSTRACT}

Begin with a short sentence or two with background on the topic. Please include a PURPOSE: describing the intent of the study. METHODS: how the study was conducted. RESULTS: findings of the study and CONCLUSION: Reference lists are not generally included.

Current published data are inconclusive regarding whether DXA body composition measures of fat, lean, and regional fat mass are predictive of other CVD risk factors. PURPOSE: To determine if DXA measures can be used in a cardiovascular risk-predictive manner to indicate unhealthy levels of circulating lipoproteins in firefighters.

METHODS: 256 male firefighters (age $=35 \pm 10$; $h t=179 \pm 6.6 \mathrm{~cm} ; \mathrm{wt}=94 \pm 16 \mathrm{~kg} ; \mathrm{BMl}=29.9 \pm 4.6$; fat mass $=27.5 \pm 10.4 \mathrm{~kg}$; lean mass $=63 \pm 7.5 \mathrm{~kg}$; gynoid\%fat $=28.7 \pm 6.5 \%$; android\%fat $=36 \pm 11.3 \%$; glucose $=85 \pm 12.9 \mathrm{mg} / \mathrm{dL} ; \mathrm{SBP}=128 \pm 9 \mathrm{mmHg}$ ) underwent an annual cardiovascular risk profile screening and DXA scan; resting BP was also measured. We drew fasting blood samples, analyzed by a clinically certified lab, to determine glucose, HDL, LDL, total cholesterol, and triglycerides. Statistics included simple statistics and Pearson's correlations. RESULTS: Table $(*=p<.01)$

\begin{tabular}{|c|c|c|c|c|c|c|c|}
\hline & & $\begin{array}{c}\text { Percent } \\
\text { Fat } \\
\end{array}$ & $\begin{array}{l}\text { Lean } \\
\text { Mass }\end{array}$ & $\begin{array}{c}\text { Fat } \\
\text { Mass }\end{array}$ & $\begin{array}{c}\begin{array}{c}\text { Android } \\
\% \text { Fat }\end{array} \\
\end{array}$ & $\begin{array}{c}\text { Gynoid } \\
\% \text { Fat }\end{array}$ & BMI \\
\hline \multirow{2}{*}{ Cholesterol } & & $.262^{*}$ & .003 & $.212^{*}$ & $.281^{*}$ & $.197^{*}$ & $.180^{*}$ \\
\hline & & .06 & .000 & .045 & .079 & .039 & .032 \\
\hline \multirow{2}{*}{ HDL } & Pearson & $-.203^{*}$ & $-.228^{*}$ & $-.253^{*}$ & $-.251^{*}$ & -.070 & $-.286^{*}$ \\
\hline & $\mathrm{R}^{2}$ & .041 & .052 & .064 & .063 & .005 & .082 \\
\hline \multirow{2}{*}{ LDL } & Pearson & $.248^{*}$ & .002 & $.206^{*}$ & $.266^{*}$ & $.191^{*}$ & $.185^{*}$ \\
\hline & $\mathbf{R}^{2}$ & .062 & .000 & .042 & .07 & .036 & .034 \\
\hline \multirow{2}{*}{ TRIG } & Pearson & $.284^{*}$ & $.166^{*}$ & $.285^{*}$ & $.322^{*}$ & .128 & $.277^{*}$ \\
\hline & $\mathrm{R}^{2}$ & .081 & .028 & .081 & .104 & .01 & .077 \\
\hline \multirow{2}{*}{ Glucose } & Pearson & $.287^{*}$ & .044 & $.271^{*}$ & $.277^{*}$ & $.238^{*}$ & $.208^{*}$ \\
\hline & $\mathrm{R}^{2}$ & .082 & .002 & .073 & .077 & .057 & .043 \\
\hline Resting & Pearson & .126 & $.201^{*}$ & $.178^{*}$ & .130 & .102 & $.176^{*}$ \\
\hline SBP & $R^{2}$ & .016 & .040 & .032 & .017 & .010 & .031 \\
\hline
\end{tabular}

CONCLUSIONS: Though the correlations were statistically significant, none of the DXA body composition measures explained a physiologically relevant portion of the variance in the CVD risk markers measured. We suggest that factors other than body fat contribute to lipid and blood pressure profiles in firefighters, a population at high risk for CVD. 